\title{
Robust Adaptive Threshold Algorithm based on Kernel Fuzzy Clustering on Image segmentation
}

\author{
Tara.Saikumar $^{1}$, B.K.Anoop ${ }^{2}$, P.S.Murthy ${ }^{3}$ \\ ${ }^{1}$ Asst Professor, Dept of ECE, CMR Technical Campus, Hyderabad, India. \\ tara.sai437@gmail.com \\ ${ }^{2}$ Lecturer, Dept of ECE, MACE, Kerala, India. \\ anoopbk2000@gmail.com \\ ${ }^{3}$ Professor, Dept of CSE, CMRIT, Hyderabad India. \\ moorthypsmegmail.com
}

\begin{abstract}
Using thresholding method to segment an image, a fixed threshold is not suitable if the background is rough here, we propose a new adaptive thresholding method using KFCM. The method requires only one parameter to be selected and the adaptive threshold surface can be found automatically from the original image. An adaptive thresholding scheme using adaptive tracking and morphological filtering. KFCM algorithm computes the fuzzy membership values for each pixel. Our method is good for detecting large and small images concurrently. It is also efficient to denoise and enhance the responses of images with low local contrast can be detected. The efficiency and accuracy of the algorithm is demonstrated by the experiments on the MR brain images.
\end{abstract}

\section{KEYWORDS}

Adaptive thresholding, Image segmentation, KFCM.

\section{INTRODUCTION}

Blind Thresholding techniques are often used to segment images consisting of dark objects against bright backgrounds, or vice versa. It also offers data compression and fast data processing [1]. The simplest way is through a technique called global thresholding, where one threshold value is selected for the entire image, which is obtained from the global information. However, when the background has non-uniform illumination, a fixed (or global) threshold value will poorly segment the image. Thus, a local threshold value that changes dynamically over the image is needed. This technique is called adaptive thresholding. Basically these techniques can be divided into region-based and edge-based thresholding. Region-based technique uses the whole image to extract the information for the threshold value computation, while edge-based technique is based on the attributes along the contour between the object and the background.

Natarajan Meghanathan, et al. (Eds): ITCS, SIP, JSE-2012, CS \& IT 04, pp. 99-103, 2012. 
For region-based technique, most of the early introduced techniques are based on the image histogram. In 1979, Otsu [2] presented a technique that considered the image histogram as having a two gaussian distribution representing the object and the background. A threshold is selected to maximize the inter-class separation on the basis of the class Variances. For the edge-based thresholding technique, the idea of applying the boundary based attributes is based on the fact that discriminant features exist at the boundary between the object and the background [3]. Thus, the edge-based thresholding technique has become more popular for exploration. Milgram [10] applied edge information to segment images by proposing "super slice" method. In this method, the edge information (gradient) is integrated with the recursive region splitting technique. The superslice method was also applied and improved in $[3,11]$.

\section{ADAPTIVE THRESHOLDING}

We adapt this technique with some optimization. The following outlines Hoover's adaptive thresholding:

1) Binarize the image with a single threshold $T$

2) Thin the thresholded image

3) Erase all branchpoints in the thinned image

4) All remaining endpoints are placed in the probe queue and are used as a starting point for tracking

5) Track the region with threshold $T$

6) If the region passed testing, $T=T-1$, go to 5)

The testing in step 6) is some constraints to guarantee the region is image segment.Very small segments that remain after thresholding will be "size-filtered". But this step is preceded by the application of morphological filter to fill the small gaps between images.

\section{Kernel fuzzy c-means clustering (KFCM):}

Define a nonlinear map as $\phi_{\bullet}^{\bullet} x \rightarrow \phi(x) \in F$, where $x \in X . X$ denotes the data space and $F$ is the transformed feature space with higher even infinite dimensions. KFCM minimized the following objective function:

$J_{m}(U, V) \equiv \sum_{i=1}^{c} \cdot \sum_{k-1}^{n} u_{i k}^{m}\left\|\phi\left(x_{i}\right)-\phi\left(v_{i}\right)\right\|^{2}$

Where $\left\|\phi\left(x_{i}\right)-\phi\left(v_{i}\right)\right\|^{2}=K\left(x_{k}, x_{k}\right)+K\left(v_{i}, v_{i}\right)-2 K\left(x_{k}, v_{i}\right)$

Where $K(x, y)=\phi(x)^{T} \phi(y)$ is an inner product of the kernel function. If we adopt the Gaussian function as a kernel function, $\quad K(x, y)=\exp \left(-\|x-y\|^{2} / 2 \sigma^{2}\right)$, then $K(x, x)=1$. according to Eq. (2.3), Eq. (2.2) can be rewritten as

$$
J_{m}(U, V) \equiv 2 \sum_{i-1}^{c} \cdot \sum_{k-1}^{n} u_{i k}^{m}\left(1-k\left(x_{k}, v_{i}\right)\right)
$$

Minimizing Eq. (2.4) under the constraint of, $u_{i k}, m>1$. We have 


$$
\begin{aligned}
& u_{i k}=\frac{\left(1 /\left(1-K\left(x_{k}, v_{i}\right)\right)\right)^{1 /(m-1)}}{\sum_{j=1}^{c}\left(1 /\left(1-K\left(x_{k}, v_{i}\right)\right)\right)^{1 /(m-1)}} \ldots \ldots \ldots \ldots \ldots \\
& v_{i}=\frac{\sum_{k=1}^{n} u_{i k} K\left(x_{k}, v_{i}\right) x_{k}}{\sum_{k=1}^{n} u_{i k}^{m} K\left(x_{k}, v_{i}\right)} \ldots \ldots \ldots \ldots \ldots \ldots \ldots . . .6 \ldots \ldots
\end{aligned}
$$

Here we now utilize the Gaussian kernel function for

Straightforwardness. If we use additional kernel functions, there will be corresponding modifications in Eq. (2.5) and (2.6).

In fact, Eq.(2.3) can be analyzed as kernel-induced new metric in the data space, which is defined as the following

$d(x, y) \underline{\underline{\Delta}}\|\phi(x)-\phi(y)\|=\sqrt{2(1-K(x, y))}$

And it can be proven that $d(x, y)$ is defined in Eq. (2.7) is a metric in the original space in case that $K(x, y)$ takes as the Gaussian kernel function. According to Eq. (6), the data point $x_{k}$ is capable with an additional weight $K\left(x_{k}, v_{i}\right)$, which measures the similarity between $x_{k}$ and $v_{i}$ and when $x_{k}$ is an outlier i.e., $x_{k}$ is far from the other data points, then $K\left(x_{k}, v_{i}\right)$ will be very small, so the weighted sum of data points shall be more strong.

The full explanation of KFCM algorithm is as follows:

\section{KFCM Algorithm:}

Step 1: Select initial class prototype $\left\{v_{i}\right\}_{i=1}^{c}$.

Step 2: Update all memberships $u_{i k}$ with Eq. (2.5).

Step 3: Obtain the prototype of clusters in the forms of weighted average with Eq. (2.6).

Step 4: Repeat step 2-3 till termination. The termination criterion is $\left\|V_{\text {new }}-V_{\text {old }}\right\| \leq \varepsilon$.

Where $\|$.$\| is the Euclidean norm. V$ is the vector of cluster centers $\varepsilon$ is a small number that can be set by user (here $\varepsilon=0.01$ ).

\section{EXPERIMENTAL RESULTS}

The output of adaptive threshold algorithm and KFCM, figure 1 is as shown below. Firstly the original image is as shown in figure (i) is transformed to a proposed adaptive threshold algorithm with the test images of the brain and original binary image, note the over segmentation. The approximate contour of white matter was got by adaptive threshold algorithm shown in Figure ii 
of Figure 1. The output of adaptive threshold algorithm is given to KFCM clustering to get the fuzzy image with fuzzy boundaries. The snooping of regions else appear as a result of the in excess of segmentation.

\section{DISCUSSIONS}

In this paper, we projected a new method to transform the algorithm. The original image was partitioned with adaptive threshold algorithm, KFCM and the controlled action of the edge indicator function was increased. The result of adaptive threshold algorithm and KFCM segmentation are as shown in Figure: 1.

Alternatively the adaptive threshold algorithm is sensitive to noise; some redundant boundaries were appeared in the candidates.

\section{CONCLUSIONS}

In this paper, we proposed an adaptive threshold algorithm, KFCM with a level set method. The results of this paper confirmed that the mixture of adaptive threshold, KFCM could be used for the segmentation of low contrast images and medical images. The validity of new algorithm was verified in the process of exacting details of images. In the future research, noise was added in images prior information on the object boundary extraction with level set method, such as boundary, shape, and size, would be further analyzed.

Figure i

Original Test Image
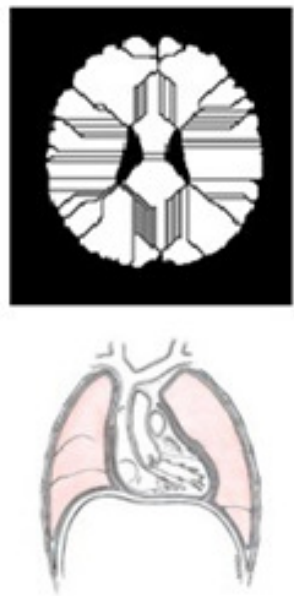

Figure ii

Adaptive Threshold test image
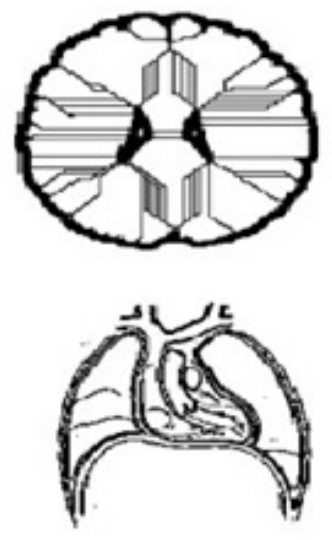

FIGURE: 1
Figure iii KFCM Segmented output
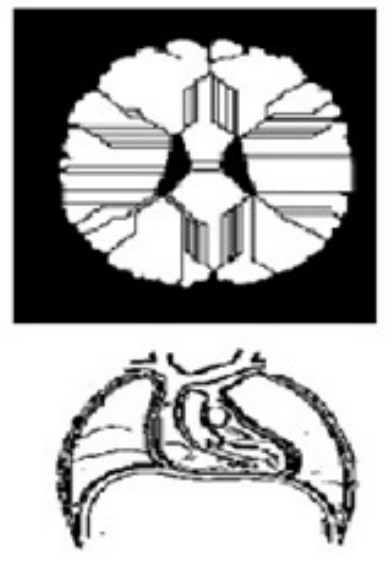

Figure $i$ are the original test images.

Figure ii are the results of Adaptive threshold images, to extracting the white matter.

Figure iii are the results of KFCM Segmented outputs.

\section{REFERENCES}

[1] A. Shio, "An Automatic Thresholding Algorithm Based On An Illumination-Independent Contrast Measure", IEEE Computer Society Conference on Computer Vision and Pattern Recognition, pp. 632- 637, 4-8 June 1989. 
[2] N. Otsu, "A Threshold Selection Method from Gray-Level Histogram”, IEEE Trans. on System Man Cybernetics, SMC, vol. 9(I), pp. 62-66, 1979.

[3] F.H.Y. Chan, F.K. Lam, Hui Zhu, "Adaptive Thresholding By Variational Method", IEEE Transactions on Image Processing, vol. 7, no. 3, pp. 468-473, March 1998.

[4] Osher S, Scthian J.A,Fronts propagating with curvature dependent speed: algorthim's based on the Hamilton-Jacobi formulation.Journal of Computational Physics,1988,pp.12-49.

[5] Malladi,R,Sethain,J., and Vemuri,B., Shape modelling with front propagation: A level set approach.IEEE Trans.Pattern Anal. Mach.Intell, 1995, pp.158-175.

[6] Staib,L., Zeng,X., Schultz,R., and Duncan,J., Shape constraints in deformablemodels.Handbook of Medical Imaging,Bankman,I.,ed., 2000,pp.147-157.

[7] D. L. Milgram, "Region Extraction Using Convergent Evidence”, IEEE Trans. on Computer Graphics and Image Processing, vol. 11 no. 1, 1979.

[8] S. D. Yanowitz and A. M. Bruckstein, "A New Method For Image Segmentation", IEEE Trans. on Computer. Vision, Graphic and Image Processing, vol. 46, pp. 82-95, 1989.

[9] J.C.Bezdek, Pattern Recognition with Fuzzy Objective Function Algorthims,Plenum Press ,New York, 1981.

[10] K.L.Wu,M.S.Yang, Alternative c-means clustering algorthims,Pattern.

[11] Mclnerney $\mathrm{T}$, Terzopouls D,Deformable models in medical image analysis: a survey.Medical Analysis, 1996,pp.91-108.

[12] S. D. Yanowitz and A. M. Bruckstein, “A New Method For Image Segmentation", IEEE Trans. on Computer. Vision, Graphic and Image Processing, vol. 46, pp. 82-95, 1989.

[13] Bezedek,J., A convergence theorem for the fuzzy ISODATA clustering algorthims. IEEE Trans.Pattern Anal.Mach.Intell., 1980,pp 78-82.

[14] L. Zhang, W.D. Zhou, L.C. Jiao. Kernel clustering algorithm. Chinese J. Computers, vol25 (6), pp. 587-590, 2002 (in Chinese).

\section{Authors}

Tara. Saikumar, received his M.Tech, degree in Digital communication Engineering from Kakatiya Institute of Technology and Science, Kakatiya University, Warangal. $\mathrm{He}$ is the university topper in his M.Tech, KITS, Warangal and also received gold medal from Dr.D.N.Reddy vice-chancellor JNTUH in his B.Tech for his excellence2004-2008. Presently working as Asst Professor in ECE, CMR Technical Education Society, Group of Institutions, Hyderabad. He his 34 paper in his credit, International journal, International and National Conferences. He is a life member of

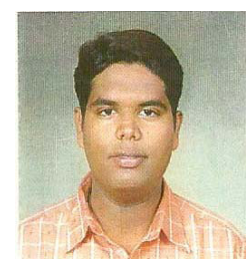
IAENG, IACSIT, and UAEEE. 